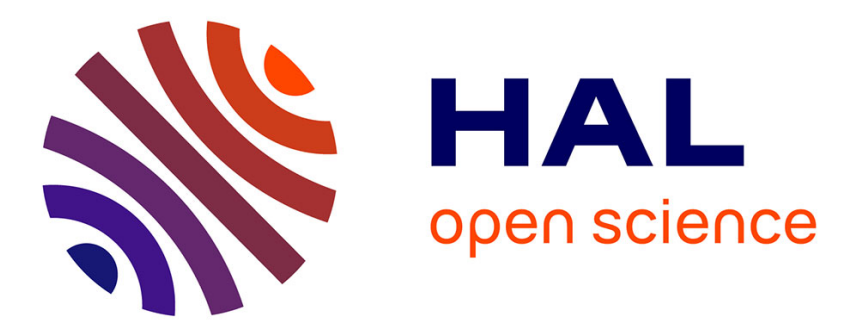

\title{
Vase peint à décor zoomorphe découvert à Roanne (Loire)
}

Pierre Vernat, Robert Périchon

\section{To cite this version:}

Pierre Vernat, Robert Périchon. Vase peint à décor zoomorphe découvert à Roanne (Loire). Gallia - Fouilles et monuments archéologiques en France métropolitaine, 1970, 28 (2), pp.207-213. 10.3406/galia.1970.2551. hal-01934504

\section{HAL Id: hal-01934504 https://hal.science/hal-01934504}

Submitted on 11 Mar 2020

HAL is a multi-disciplinary open access archive for the deposit and dissemination of scientific research documents, whether they are published or not. The documents may come from teaching and research institutions in France or abroad, or from public or private research centers.
L'archive ouverte pluridisciplinaire HAL, est destinée au dépôt et à la diffusion de documents scientifiques de niveau recherche, publiés ou non, émanant des établissements d'enseignement et de recherche français ou étrangers, des laboratoires publics ou privés.

\section{(이) $\$$}

Distributed under a Creative Commons Attribution - NonCommercial - NoDerivatives $\mid 4.0$ 


\title{
Vase peint à décor zoomorphe découvert à Roanne (Loire)
}

\author{
par P. VERNAT ef R. PÉRICHON
}

Depuis 1966;, une expropriation de terrain, en rue d'un nouveau tracé de la route nationale 7 à travers Roanne, a permis d'entreprendre des travaux archéologiques ${ }^{1}$. Parmi les nombreuses découverles effectuées. il en est une qui retient notre attention; il s'agril d'un vase peint d'une époque lardive de la Tine qui porte un décor zoomorphe. Ia rareté de tels vestiges et l'expression du réramiste gaulois sont d'un grand intérêt et nous incitent à présenter une étude de cet objet et de son contexte.

Il s'agit de la périphérie du villagre de la Tine ou village gaulois ${ }^{3}$ situé sur les terrains

I Il sagil de terrains situes entre la rue de Charlieu, la rue Gilbertes et la place Louis Flandre. Ces travaux ont ite effectues sous la direction de .I. Jean Poncer. professeur d'histoire et de grégraphie an Lycere JeanPuy, a Roanne. Plusieurs éludes de Jean Poncel apportent des précisions sur les travaux entrepris: cf. Les Fouilles de la rue Cilberles, dans Cahier d'archéologie régionale, publication du Groupe de recherches archéologiques de Roanne, $n^{\circ} 7,1968 \mathrm{ct}$, Les recherches archéologiques à Roanne, dans Eludes Foreziennes, I, Mélange's, 1968, p. 25 a 40.

2 Nous utilisons la chronologie da Dechelette Manuel, second lye du fer ou époque de la Tène, edit. de 1927, p. $41 \times$. En ce qui concerne la periode de la Tene en Forez on an Roannais, cette chronologie peut ètre utilisere, avec cette nuance que la derniere phase Tene Ill, que Dechelette fait aller de loo a la premiere anné de notre ère, doit être interrompur à - 50, anné pendant laquelle la Gaule est devenur province romaine.

3 Iont lielude a iti conduile, jusqu' ('n juin 1969, par II. TAbbe Bessou, professeur a l'Institution saint-paul. de l'Institulion saint-Paul'. L'occupation semble continue, depuis la période vauloise jusqu'au début du $11 \mathrm{I}^{\mathrm{e}}$ siecle avec des phases de plus ou moins grande intensité. Il s'avère que les phases de grande intensité dispersent les traces des occupations antérieures mais certaines fosses profondes. areusées par les premiers habilants, se sont conservées intactes jusqu'a nous ${ }^{5}$.

Les fragments de vase peint que nous présentons ont été découverts dans une de ces fosses, située dans les carrés JK 11 el J 12 (plan, fig. 1$)$ du chantier (; $1^{6}$.

Creusée dans le substratum sableux, la fosse apparaissait hémisphérique. On pouvait l'observer à partir du niveau -..-1.90 m. Le fond

4 A la suile d'un changement d'organisation, lancienne Institulion Saint-Joseph est devenue l'Instifution Saint-Paul. Ln certain nombre de publications font itat de decouvertes à l'Institution Saint-Joseph. Pour cefte raison, nous avertissons le lecteur que ces deux appellations designent le mème établissement at le mêmo site.

5 Selon J. Poncel, la plupart de ces fosses, tries variables par leurs formes et par leurs dimensions, sont trop petites et trop profondes pour être des fonds de cabantes. (f. Les recherches archéologiques à Koanne..., p. 29. On peut trouver l'etude détaillee de l'une de ces fosses dans la note publice par R. Pronchox el J. CABOTSE, Analyse d'une fosse à déblais de l'Instilulion Saint-Joseph à Roanne (Loire), dans Cellicum IX, 1964, p. $189 \mathrm{et} \mathrm{s}$.

6 Ies differents travaux effectués sur le site de Gilbertès sont designés par les lettres G., suivies d'un chiffre: $1,2,3,4$, elc.., désignant les chantiers et les sondages. 


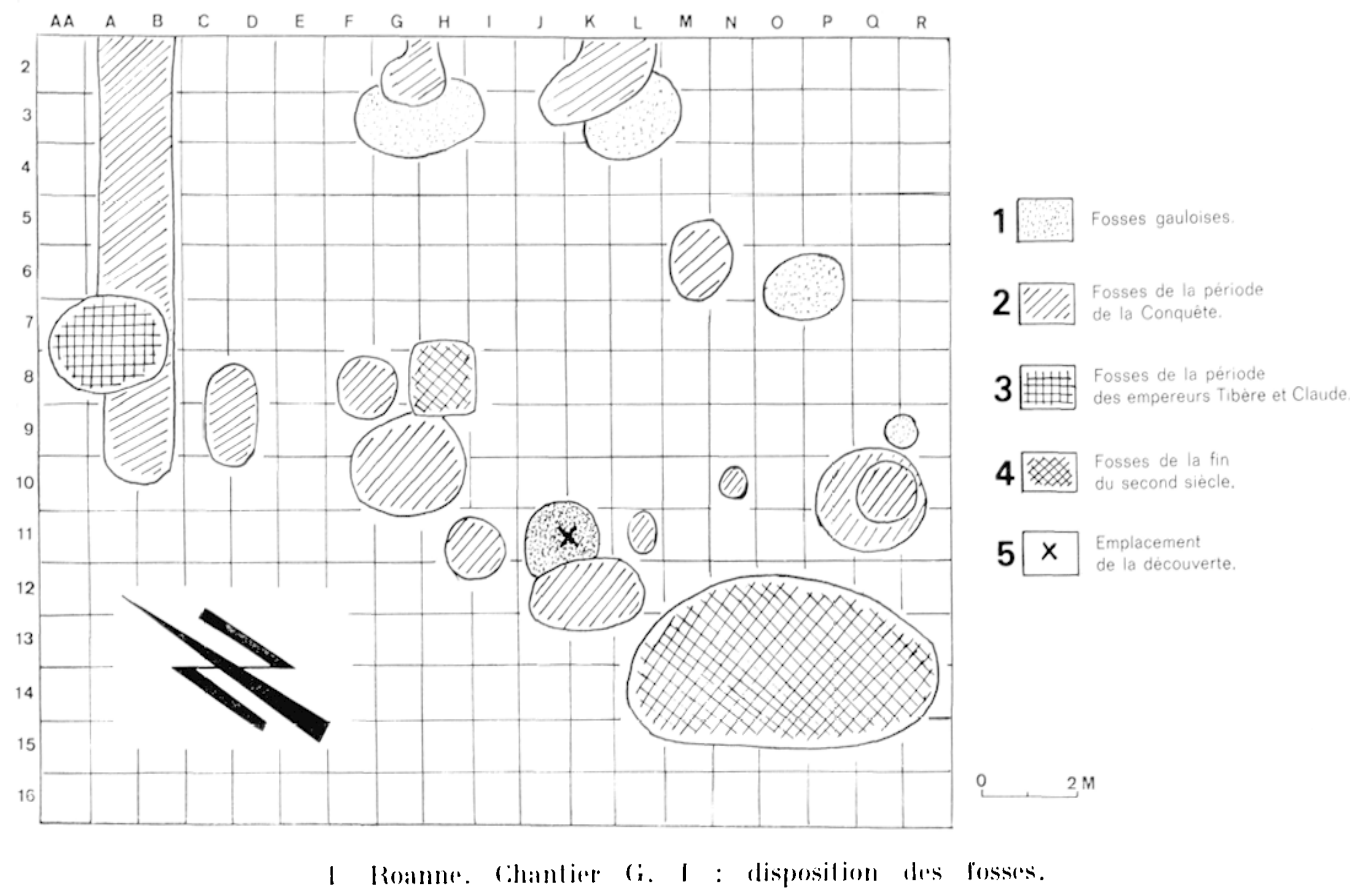

alteignail $2,80 \mathrm{~m}^{7}$. Son remplissage etait constilue par de la terre grasse contenant de nombreux débris de charbons de bois. des os en partie ralcinés el de la céramique fragmentée. les éléments de datation rontenus dans cetle fosse sont rares: on observe. en mème temps qu'un lot homogine de céramiques domestiques: communes de fabrication rustique (tig. 2. :3. 4 et 5). quelques tessons de vases appartenant a des imilations de créramiques campaniennes (fig. 6). Par comparaison aver les céramiques d'autres grisements ${ }^{8}$ il semble que le creusement de celle fosse, ainsi que les restiges qu'elle contient, remontent à la périorle de La Tóne III. rans une phase antérieure à la conqueite.

Forme. Il s'agit d'un vase de forme haute que l'on peul désigner par le lerme llacon. La panse est arrondje et son renflement maximum se situe environ aux deux liers supérieurs du récipient ifig. $7 . n^{\circ} 1$. Le col est légerement basé. Le pied est sépare de la panse par un

7 Les profondeurs $-1,90 \mathrm{~m}$ et $-2 . \times() \mathrm{ml}$ sont mesurées par rapport all niveall actuel de la rue Gilberties.

* En particulier, celuj de l'usine à gaz, de: Bàle.

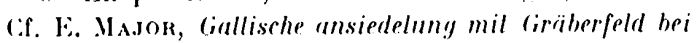
Basel, Bàle, 1940. rétrécissement asse\% marqué. Le fond n'est, pas plat mais légirement ionvexe. Dans les formes de ce lype qui se rencontrent en Gaule. les dimensions ne sont pas ronstantes et les proportions mimes se modifient. Ies exemples nous sont donnes par le remarquable vase de la sepulture 1 d'Aulnat' et celui. trés élégant, dérouvert par J.-R. Terrisie à Corent ${ }^{10}$. Le Iont-Beuray a livré quelques exemplaires de retle forme ${ }^{11}$.

Ce type de vase forme 2 de la classification de R. Périchon ${ }^{11}$ bis nest pas sans evoquer reux de La Tine ${ }^{12}$ de la Marne et des

9) I. Informations, dans diallia, XXV, l967, p. $306-3307$.

11) K. Trennssti, lases gaulois du debul de la Conquete romaine decouverts sur le plaleau de Corent

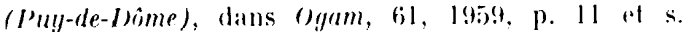
l.d diamele de ce vase est de $0,120 \mathrm{~m}$, pour $\mathrm{mm}$ hatuleur de $0,300 \mathrm{~m}$.

11 F. ot X. THombn, Fonilles du Mont-Benvay (ancienne Bibracte), Album, saint-Etimme, 1899, pl. 3.

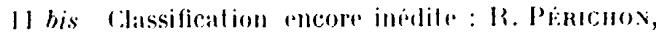
la reramique peinle guuloise of gallo-romsine dans le Centre de la tianle, thise de 3" cycle soutenue a lat Farulti de citemont-Ferrand, 1967. Voir la pl. 2.

12 1. Dronsasox, Bullelin de la sociele des Antiquates de France, 191:2, p. 279 a $396 \%$. 


\section{7}

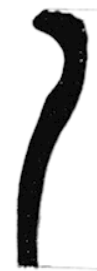

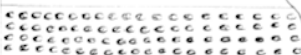
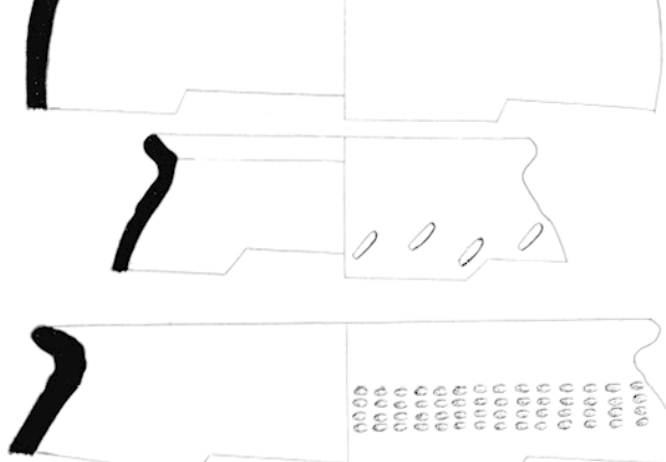

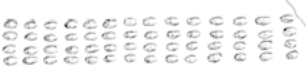

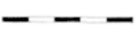

2 Ciramiques domestigues commumes : fragments de vases ovoüdes ormis.
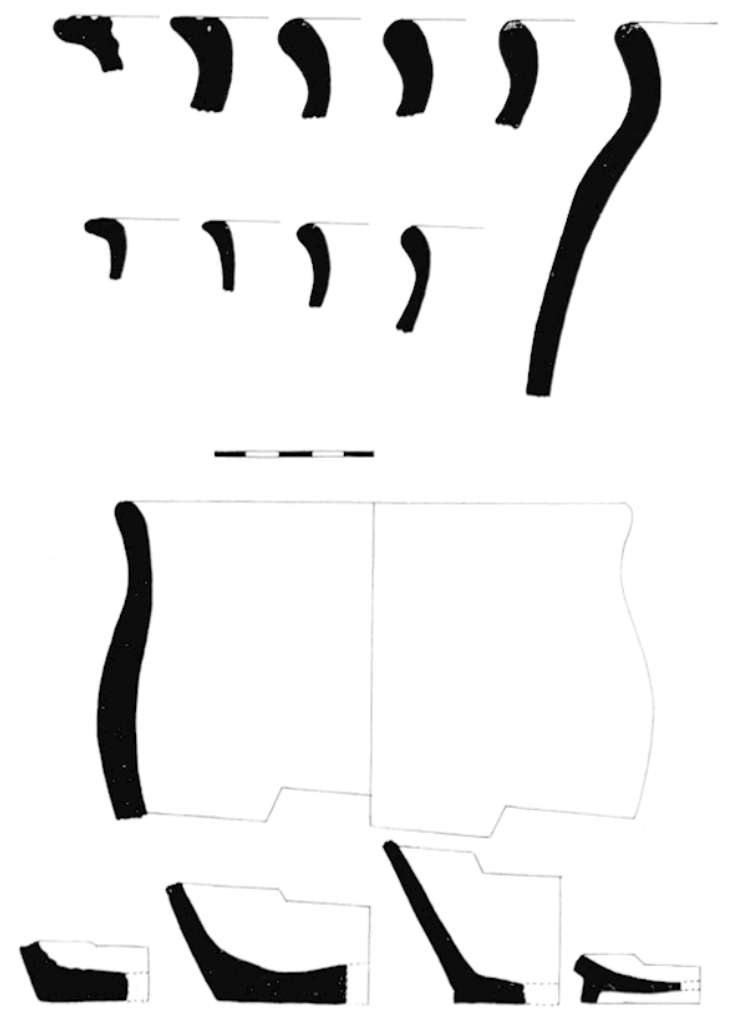

1 Cinamiques domestiques commumes : cols ol fomds de vases ovoidles, sauf en bas at ia droile, un fond de coupe.
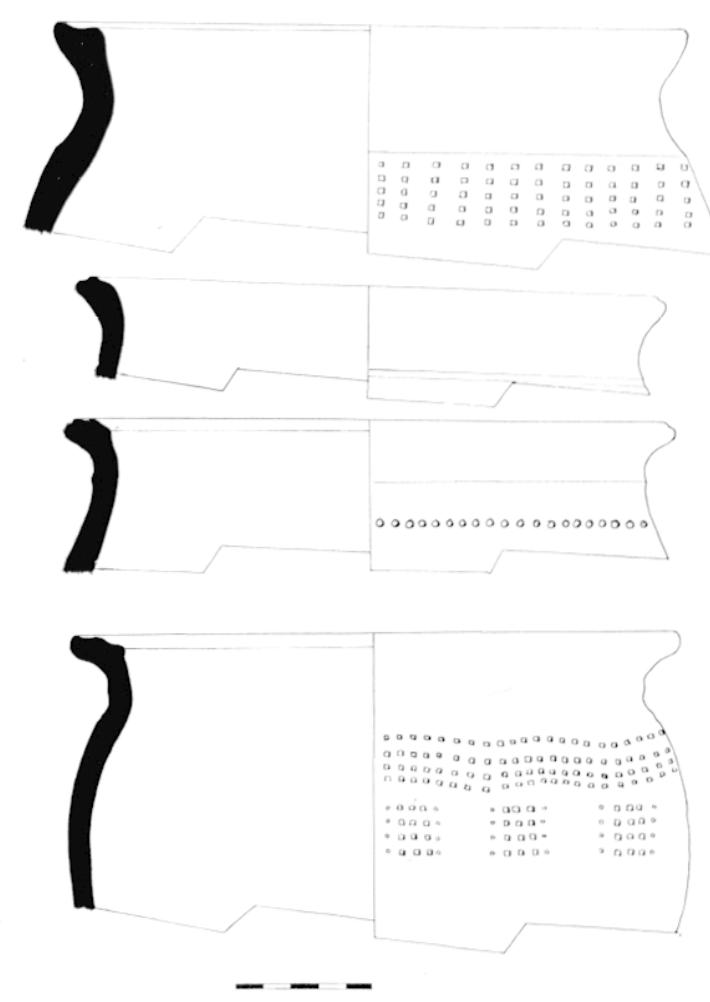

3 Cirimigues domestiques communes : fragments de vases ovoïdes ormis.
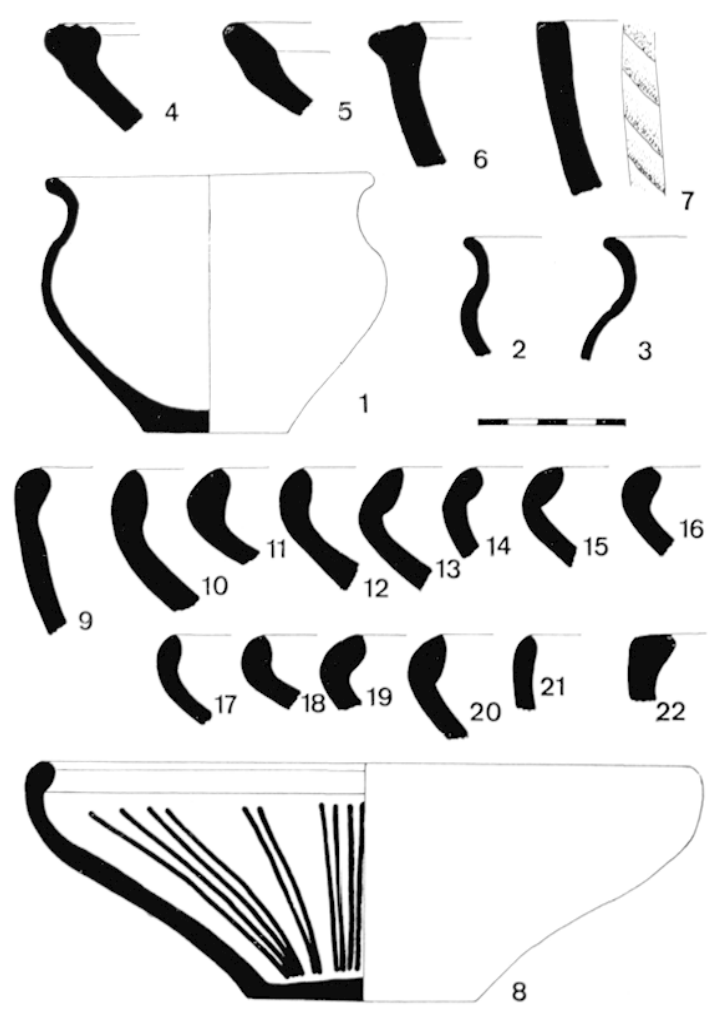

5) Ceramiques domestifues communes : no 1 : petit vase rustigue; $n^{0 \times} 2$ et 3 : fragments appartenant all mème type de recipient; nos 4 à 7 : rebords décuelles; $110 x:$ iccuelle à rebord rentrant decorée au lissoir ; $n^{\circ \times} 9$ a :2.2 : profils de vases du même: typr. 

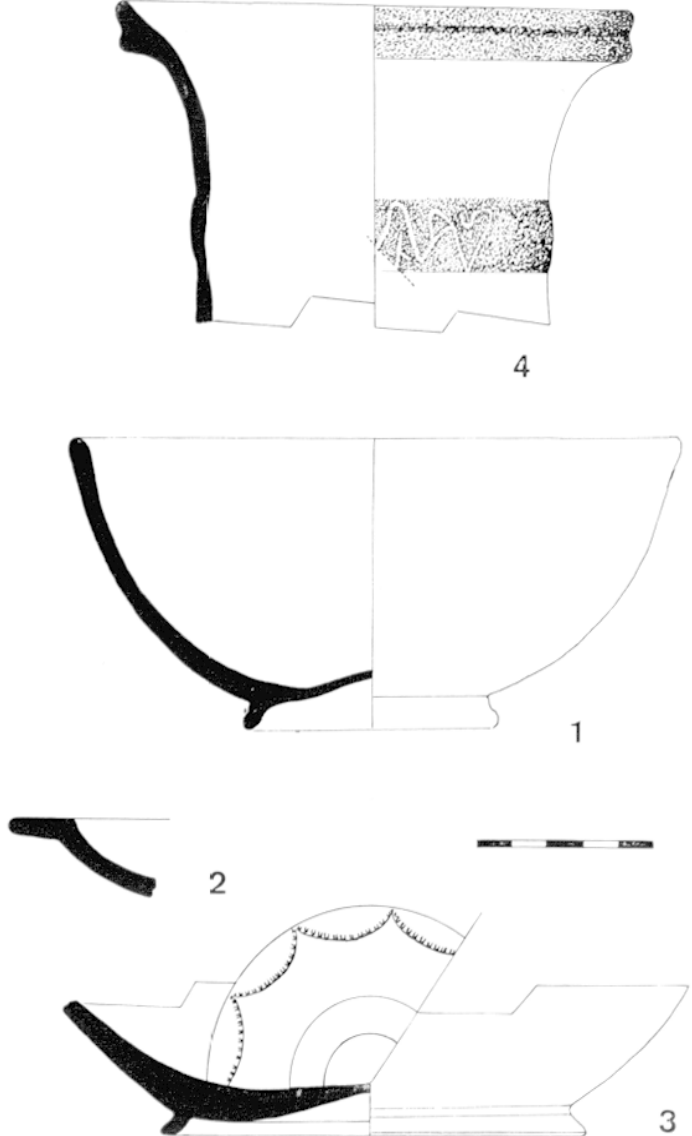

6 now 1 a 3 : implations de ceramiques campantenues: nol : coupe: $n^{\circ}: 2$ : fragment de bord: $n^{\circ} 3$ : fragment de coupe decorio ; no 4 : fragment de ceranique peinte presenlanl un graflito.

Ardennes. C'est la forme la plus courante et la plus ancienne qui se rencontre a Aulnat. a Corent el a l'Institution saint-Joseph. a Roanne ${ }^{13}$, dans des niveaux correspondant a une occupation antérieure a la Conquele. fion usage se poursuit cependant apres la Conquete. mais sous un aspect moins élégant et en nombre restreint.

Technique de fabricalion. Le vase de (iilbertis a probablement éle tourné. comme semblent en témoigner des stries tris fines, bien visibles. a l'intérieur. La surface interne présente une série d'irrégularités dans l'épaisseur des parois

13 R. PÉrichox ot J. Cinborst, Analyse d'une fosse à deblais de l'Instilution Sainl-.Joseph, à Roanne, dans Cellium IX, 1963, pl. 14, fig. 14/4, coramiqur. peinte du niveau 6. formant une série de bosses qui sorganisent horizontalement. La pàle est grossiere. I n examen a la lunetle binoculaire montre qu'elle contient quelques gros grains de quartz ou de feldspath. tres dispersés, ainsi qu'une quantité importante de particules de mica.

la coupe mont re que la cuisson a été elfectuée a l'aide d'un four plus ou moins primilif rar l'oxydation, bien visible pris des surfaces, n'a pas alteint le corur de la paite qui est resté rris. Ce fait a déjá été observé sur des céramiques de meme type provenant de l'Institulion saint-.Josephh' et d'Aulnat ${ }^{15}$.

Ornemenlation. Deux modes d'ornementation sont utilises : le lissage et la peinture.

Le lissage. In souci de finition a fait exécuter au polier ou au décorateur des traits de lissage visibles sur le pied du récipient. Ils se présentent sous la forme de bandes asse\% grossieres. a peu pris horizontales el paralleles (fig. 8. $1^{\circ} 2$ 2) Le col est enlierement lissé : ce lissage recouve méme l'inlérieur de la live sur une hauteur de I rom.

La peinture. On distingue deux formes de peinture : relle qui sert de fond el les motifs zoomorphes :

la peinture de fond. Il s'agit d'un engobe rougere. dorigrine minerale, disposé avant, cuisson. Son aspect est uniforme et régulier. In lissage, probablement postérieur à la ruisson. lui donne. malgre la couleur sombre. un certain brillant. Cet engobe sert de support aux dessins \%oomorphes;

les motifs zoomorphes. Ils sont raisemblablement peints aver des jus végétaux et représentent une théorie d'animaux orientés a droite (fig. 7, no 1 et fig. 9). Ces animaux sont au nombre de quatre sur le pourtour du récipient. Ils ont été peints arec un pinceau probablement asse\% reche, romme semblent l'indiquer les stries observées sur rertains remplissages. Les contours sont précis. d'un trail épais de 2 a $3 \mathrm{~mm}$ de large, de couleur

14 Observations des auteurs.

1) V. Informalions, dans (iallia, XXV, 1967, p. $306 \mathrm{j}-3077$

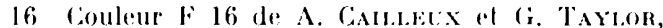
Code expolaire des couleurs des sols, Boubee, 19; $x$. 

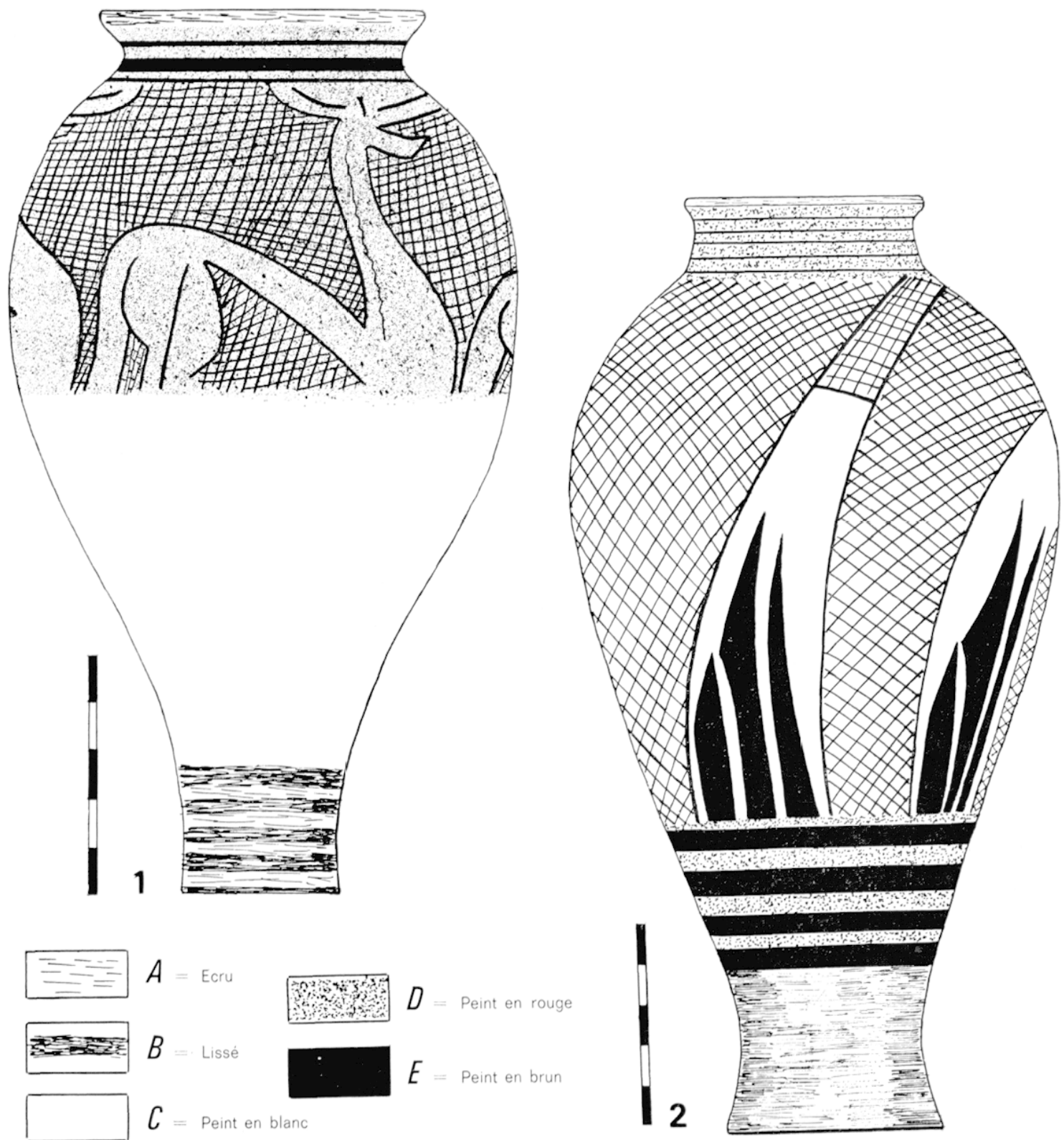

7 no 1 : restitution de la forme el du décor du vase peint de Gilbertes : no 2 : comparaison d'une stvlisation très poussée sur un vase de l'Institution Saint-Joseph à Koanne. Dapries un dessin de .11. Bessou!.

brune ${ }^{17}$. I.es interstices sont garnis avec des traits entrecroisés formant un lin treillis irrégulier par endroits. I.es animaux sont

17 Couleur J 12 ou J 22 du code expolaire (op. cil., note 16 . La designation précise du code expolaire est, pour J 12, "rouge: sombre "et, pour J 22 "brun rouge fonce ". En fait, a l'origine, la couleur devait ètre brun gris ou brun fonce, mais son alteration laisse apparaitre la couleur du fond. représentés sur la partie la plus renflée de la panse. Leur corps est penché en avant. Ia croupe. trés exagéréce par rapport au tronc, se termine par une queue large. La cuisse droite est disposée normalement par rapport à la croupe el se prolonge par une patte mince. Ia ruisse grauche est décalée vers la droite; une cassure du récipient ne nous permet pas de voir la palle qui s'y rapporte. le ventre tris 

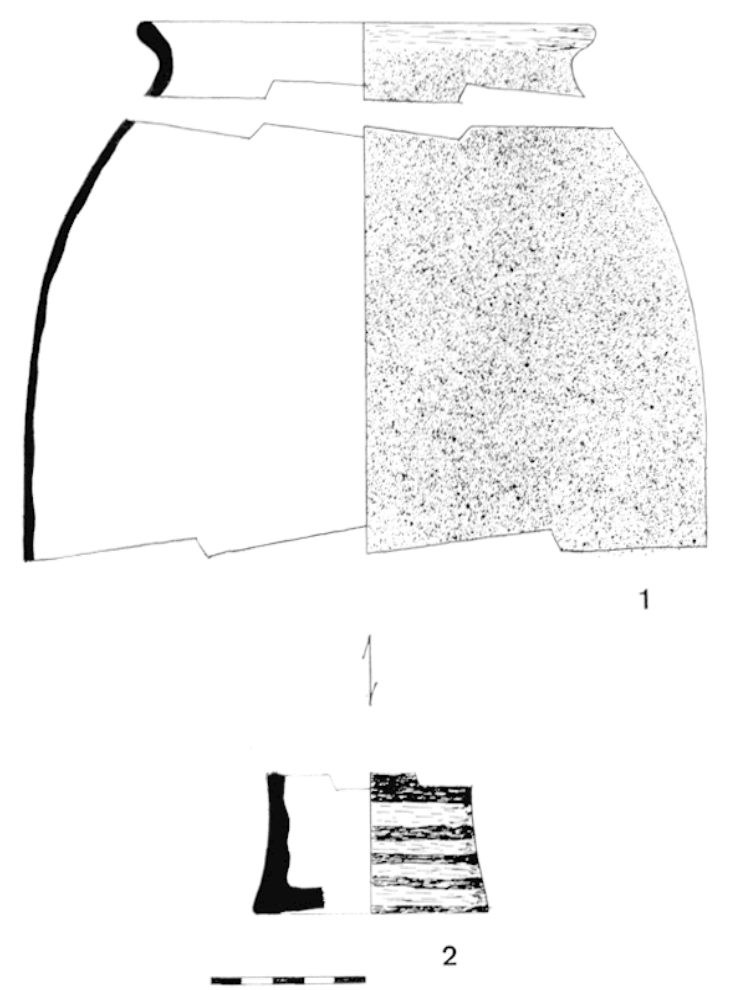

s liestilution du col, de la panse ol du pied dil vase peinl.

eflilé se lermine par un poilrail volumineux. Le cou, élevé en forme de col de cyone, est gracieux. In trail en zig\%ag. sur le cou, voudrait symboliser une criniere? I.a reconstitution de la tête est considérablement gênée par la fragmentation du vase : de chaque coté du crâne, on observe deux larges et grandes oreilles et, sous l'une d'elle, à droite, dans une disposition rurieuse, un trait courbe symbolise le museau. Pour celle meme raison, les paltes antérieures manquent.

Quel animal a voulu représenter le potier? Beaucoup d'indices laissent supposer qu'il s'agit de chevaux : arriere-train, queue, criniere. poitrail. Cependant, la disposition curieuse de la tête, et plus particulierement celle des oreilles, evoque une sorte de gazelle ou de biche. Or, les deux types d'animaux se rencontrent sur des vases peints de Roanne ${ }^{18}$. Iartiste

18 Abbe Marius Brssoc, La polerie peinle gauloise ì Roanne (Loire) an Ier siècle avanl .J.-C., dans Ogam, XIX, 1967, p. 116-117, pl. 47, fig. 3 at pl. 4x, fig. 21 et 22 . a-l-il ite conduil par une sorte de syncrelisme pour evorguer les deux animaux en une seule figure? Xous ne somserivons quaver prodenere i) relle hypothese.

la découverte de ce récipient vient s'ajouler aux deux aulres vases a décor zoomorphe connus et recueillis a quelques dizaines de mitres de la. Ies aulres vestiges découverts en meme lemps : reramiques domestiques de fabrication grossiere, imitations de céramiques campaniennes, laissent supposer une fabrication un peu antérieure à la Conquète. Cette derniere observation rejoint celle de M. l'abbé Bessou qui attribue aux fabrications de la fin de l'Indépendance les réramiques peintes à décor zoomorphe découvertes par lui à l'Institulion saint-Joseph ${ }^{19}$. La question se pose de l'origine de ces reramiques. Nous pouvons, sans grand risque d'erreur, avaneer qu'il s'agrit de fabrications locales. Nos raisons sont les suivantes : il est rertain que les argiles de Roanne ont été utilisées dans l'Antiquité; nous en avons la preuve formelle par la découverte de fours de potiers gallo-romains a Roannev; de plus, il est possible de déceler une évolution locale des formes et des décors. tout partirulierement des dérors zoomorphes. Il est aussi possible de déceler une évolution de la stylisation des animaux qui, dans certains cas, peut iltre poussée a l'extrime par le décorateur. comme rela semble itre le cas sur un vase découvert a l'Institution siaint-Joseph que nous reproduisons (lig. $7, n^{0} 2$ ), a litre de comparaison.

De la a supposer, comme le prétend J.-.J. Jully. que le Forez a été le creuset d'une stylistique ornementale ${ }^{21}$. c'est aller vile en ouvrage : il serait d'abord nécessaire de découvir les sources d'inspiration des réramistes.

Jans l'élat acluel des recherches a hoanne. res sources restent à découvrir mais il ne serait pas surprenant que les annees a venir nous

19 Ibid., p. $114 \times 11 \%$.

20 J. Poxcer, Fours de poliers gallo-romains de Roanne, Loire, dans la Revue Archeologique du Cimlre, n०22, 1967, p. 132 el s.

21 J.-J. Jti.y, Eitude genérale sur les vases ovoïdes el globulaires à engobe blanc el blanc gris de La Tine et du début de nolre ire ( (iaule centrale el méridionale), dans les Cahiers Rhodaniens, VIII, 1961, p. .23 a 6:2. 

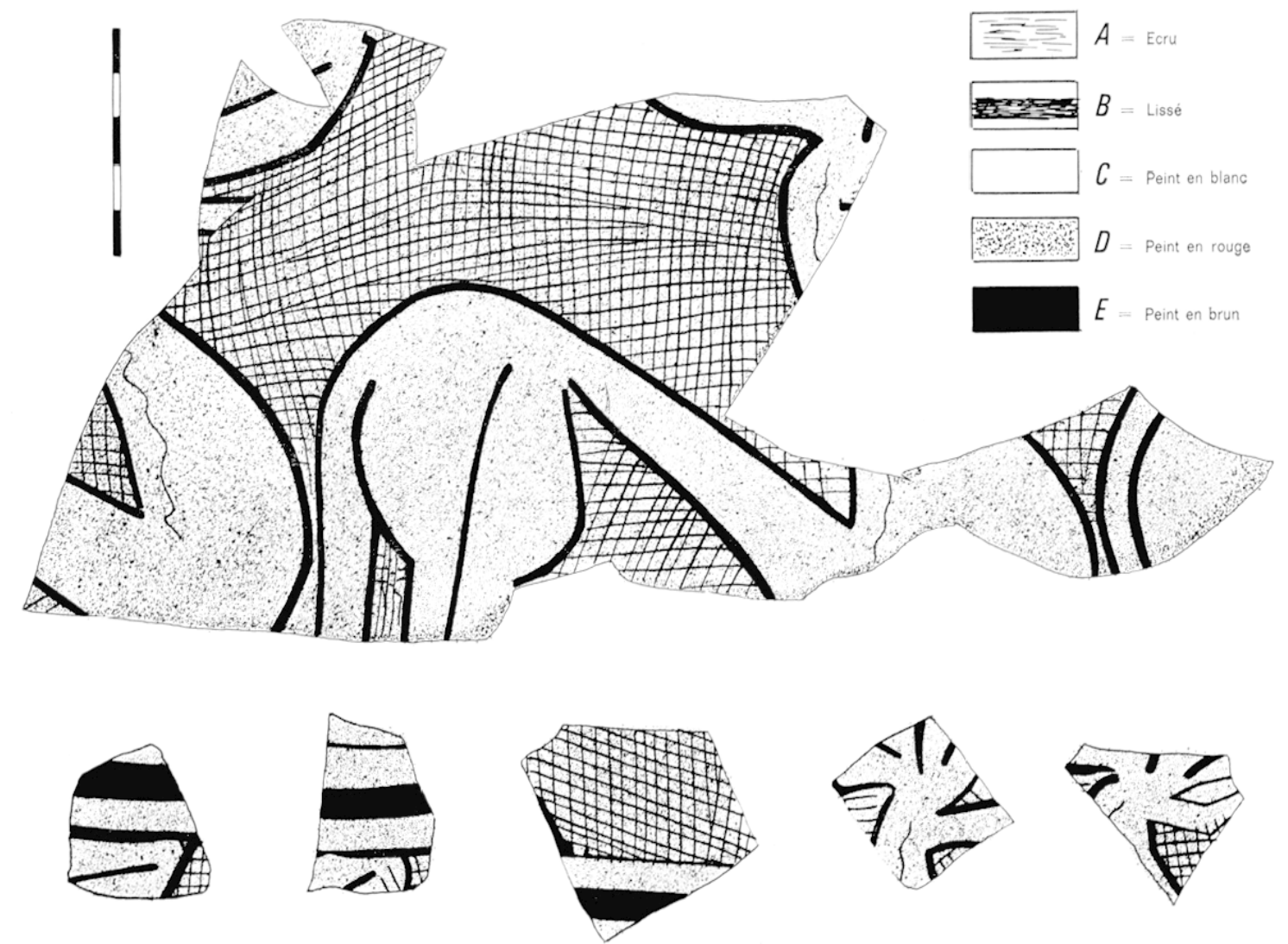

(4) Fragments du vase print rencueillis.

permettent d'observer des phases d'occupation antérieures a celles que nous venons d'évoquer et qui livreraient des céramiques peintes. Cette supposition est avancée pour la raison simple que cerlains vases peints découverts a Roanne el ornés de molils zoomorphes sont décorés selon une lechnique que nous observons sur les vases peints de la Marnez2, res derniers

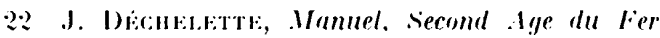
ou époque de la Time, idit. de 19:7, p. 971, fig. 661, vase de Betheng Marne;. découverts dans un contexte de Ia Tène I, soit environ entre 450 et :300 avant notre ire. la mème observation a été effectuée dans le voisinage du grisement d'Aulnal, en Auvergne ${ }^{23}$ dont la réramique peinte bénéficie probablement des mimes sources d'inspiration.

\section{P. Versat el R. PÉrichox.}

:33 (i. Foc RNil: Découverles celliques et galloromaines ia Aulnal, fiandaillal, Ilerbel, Sarlieve, dans la Rerne d'.1urergne, $81, n^{\circ} 4,1967$, p. 272 et 273 . 